\title{
Staphylococcus hyicus
}

National Cancer Institute

\section{Source}

National Cancer Institute. Staphylococcus hyicus. NCI Thesaurus. Code C86763.

A species of facultatively anaerobic, Gram positive, cocci shaped bacteria in the phylum Firmicutes. This species is positive for catalase, caseinase, hyaluronidase, and alkaline phosphatase, and negative for coagulase, oxidase, and urease. It can ferment sucrose, mannose, ribose, lactose and trehalose but not arabinose, cellobiose, maltose, glycerol, mannitol, turanose, xylitol or xylose. S. hyicus is a zoonotic pathogen. 